\title{
Investigation of the frequencies of free oscillations of the above-ground main pipelines depending on the type of fastening
}

\author{
Vladimir Sokolov ${ }^{1}$, Igor Razov ${ }^{1, *}$, Evgeniy Koinov ${ }^{1}$, and Alexander Korkishko ${ }^{1}$ \\ ${ }^{1}$ Industrial University of Tyumen, Volodarskogo str. 38, 625001, Tyumen, Russia
}

\begin{abstract}
In this paper, we solve the problem of free bending vibrations of a thin-walled gas pipeline of large diameter laid above the ground on supports. The gas pipeline is considered as a cylindrical shell subject to the action of a stationary internal working pressure, the parameter of the longitudinal compressive force. The frequencies of free oscillations and the influence of parameters on the gas pipeline are compared with different versions of the supports.
\end{abstract}

\section{Introduction}

Pipeline transportation is an important component of the fuel and energy complex of the Russian Federation, it has a length of tens of thousands of kilometers from the point of production to consumers. Many projects are currently being implemented to build new and upgrade existing pipelines. They are made of modern thin-walled pipes with a diameter of more than $1000 \mathrm{~mm}$. Depending on the region, climatic and geological conditions, there are different types of technological solutions when laying pipelines, for example, they distinguish: underwater, above-ground, above-ground, underground, and semi-subterranean [2]. For each type of gasket there are specific features of calculation and collection of loads, accounted for during operation.

\section{Problem}

Overhead laying of pipelines is one of the most frequently used in connection with its uniqueness and ease of construction. The most rational use of it in areas with rugged terrain, a large number of rivers, lakes, etc., in areas of landslides, mine workings, on subsidence permafrost soils and in other difficult conditions.

When installing overground pipelines, various versions of the pipeline supports are used for support, this depends on the diameter of the pipeline, its operating mode and the loads acting on the pipeline. There are four main types of fastening, namely:

- $\quad$ sliding - these supports are used in regions where it is necessary to compensate for seasonal temperature changes, they allow the whole system to be stable and stationary, and also compensate for longitudinal displacements;

\footnotetext{
${ }^{*}$ Corresponding author: Razov1990@yandex.ru
} 
roller support - these supports are used when it is necessary to ensure the ductility of the pipeline and in areas where it is necessary to create the smallest frictional force;

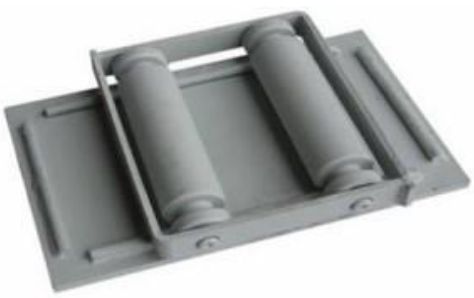

Fig. 1. Sliding support.

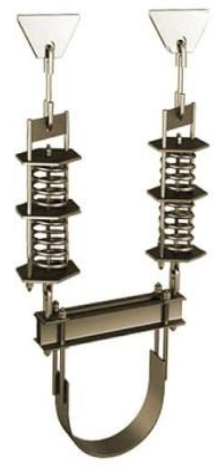

Fig. 2. Suspended support.

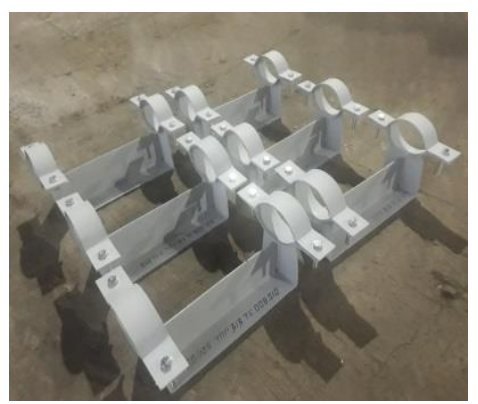

Fig. 3. roller support.

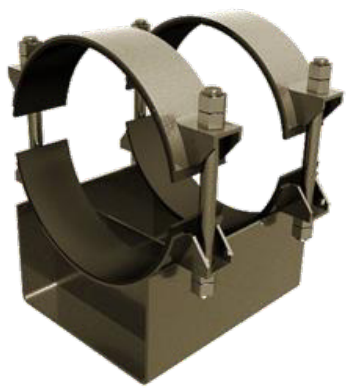

Fig. 4. Rigid support. 
- $\quad$ suspended - distinguish between spring and rigid suspension supports, rigid ones are used for pipes of diameters from 25 to $600 \mathrm{~mm}$, and spring ones for pipes with diameters from $150 \mathrm{~mm}$ to $1400 \mathrm{~mm}$. The work of the spring support is comparable with the operation of the sliding support and allows the spring support to compensate for variations in the length of the main due to the differences in both operating and climatic changes in temperature;

- $\quad$ rigid - these supports are mainly used in the northern regions of the country with thermal insulation of pipes. Extremally low temperatures have a negative effect on the condition and integrity of the structure and make it unfit for use. As already mentioned, such support elements are used in those parts of the engineering structure where it is necessary to compensate for the load on it, protect against deformations, later temperature changes, vibrations and hydraulic shocks.

At present, thanks to its efficiency, thin-walled main pipelines have found wide application in the oil and gas industry. One of the important design problems is dynamic calculation, since the linear part is subject to significant dynamic influences and vibrations. There are various calculation methods, regulated by SNiP 2.05.06-2010 and other normative documents, where the frequency of free oscillations is determined. Depending on the type of pipeline (thin-walled or thick-walled), such calculation schemes as the rod model and the thin-walled cylindrical shell model.

\section{Description of studies}

For main pipelines there is a large number of solutions, they were received by V. Bolotin. (1), Feodosiev V.I. (2), as well as they are fixed in the norms of PNAE G-7-002-86 (3), however, according to these formulas, one can not take into account the influence of the internal working pressure on the natural frequencies of vibrations and the deformation of the cross section, which is characteristic of real thin-walled pipelines.

$$
\begin{gathered}
\omega_{i}=\frac{i^{2} \cdot \pi^{2}}{l^{2}} \sqrt{\frac{E I}{\left(m+m_{0}\right.}\left(1-\frac{F l^{2}}{i^{2} \pi^{2} E I}\right)}, \\
\omega_{i}=\frac{\alpha^{2}}{l^{2}} \sqrt{\frac{E I}{m+m_{0}}}, \\
\omega_{i}=\frac{i^{2} \cdot \pi^{2}}{l^{2}} \sqrt{\frac{E I}{\left(m+m_{0}\right.}\left(1-\frac{V^{2} m_{0} l^{2}}{i^{2} \pi^{2} E I}\right)},
\end{gathered}
$$

Therefore, for the calculation of thin-walled gas mains, the theory of cylindrical shells should be applied. According to this theory, the pipeline is represented as a cylindrical shell of finite length $L$, the radius of the median surface $R$, and the wall thickness $h$. In this theory, in contrast to the core, the influence of the internal working pressure $p 0$, the effect of the nonuniform elastic backing of the soil on the cross section and the longitudinal compressive force $\mathrm{F}$.

However, using different types of supports, we provide various fixations on the boundaries of the shell, which affects the frequencies of free oscillations of the pipeline, VG Sokolov. in his work [3] converted the formula 4 and obtained formulas for 3 versions of the location of the pipeline supports.

$$
a^{2}{ }_{\mathrm{l}}=\frac{I_{i n}^{I V}+h_{v}^{2} m^{4}\left(m^{2}-1\right)\left(m^{2}-1+p^{*}\right) I_{i n}+2 \varepsilon_{0}^{*} h_{v} m^{4} I_{i n}^{I I}}{R h h_{v}^{2} \rho^{*}\left[\left(m^{2}+m^{4}\right) I_{i n}-I_{i n}^{I I}\right]}
$$


Option 1. Both ends of the shell are hinged.

Let us assume that the end sections of the shell are fixed by flanges. This fixation assumes that the rigidity of the flange is large in its plane and does not have rigidity from the plane. Flanges do not allow the movement of the edges of the section along the normal to the middle surface and displacements in the circumferential direction. In addition, the supports do not allow the movement of the edges of the shell along the longitudinal axis $\xi$.

In this case, the boundary conditions at the ends of the shell are determined by the following expressions:

$$
\begin{gathered}
\text { at } \xi=0 \text { and } \xi=l=\frac{L}{R} \quad w=v=0 ; T_{1}=M_{1}=0 ; \vartheta_{2}=0 \\
\text { At } \xi=0 \text { and } \xi=l=\frac{L}{R} \quad w=v=0 ; \frac{\partial u}{\partial \xi}=0 ; \frac{\partial^{2} w}{\partial \xi^{2}}=0
\end{gathered}
$$

The fundamental function corresponding to this bind ing is adopted:

$$
f_{n}(\xi)=\sin \frac{\lambda_{0}}{l} \xi \text {, at } \lambda_{0}=n \pi, l=\frac{L}{R}, n=1,2 \ldots
$$

Integral expressions with allowance for this type of binding will be written in the form:

$$
\begin{gathered}
I_{n n}=\int_{0}^{l} f_{n}(\xi) f_{n}(\xi) d \xi=\int_{0}^{l} \sin ^{2} \frac{\lambda_{0}}{l} \xi d \xi \\
I_{n n}^{I V}=\int_{0}^{l} f_{n}(\xi) f_{n}^{I V}(\xi) d \xi=\frac{\lambda_{0}^{4}}{l^{4}} \int_{0}^{l} \sin ^{2} \frac{\lambda_{0}}{l} \xi d \xi, \\
I_{n n}^{n}=\int_{0}^{l} f_{n}(\xi) f_{n}^{\prime \prime}(\xi) d \xi=-\frac{\lambda_{0}^{2}}{l^{4}} \int_{0}^{l} \sin ^{2} \frac{\lambda_{0}}{l} \xi d \xi=-\frac{\lambda_{0}^{2}}{l^{2}} I_{n n}^{*} I_{n n}^{*}=I_{n n} \\
I_{\text {in }}=\int_{0}^{l} f_{i}(\xi) f_{n}(\xi) d \xi=0, \text { at } i \neq n .
\end{gathered}
$$

Substituting (5) into (4) with regard to (8), after the transformations, we obtain the expression for the square of the circular frequency of the free vibrations of the shell, first dividing the numerator and denominator by the parameter $h_{v}^{2}$ :

$$
\begin{gathered}
w_{\min }^{2}=\frac{\lambda_{v}^{4}+m^{4}\left(m^{2}-1\right)\left(m^{2}-1+p^{*}\right)-\lambda_{n}^{2} m^{4} 2 \varepsilon_{0}^{*}}{R h \rho^{*}\left(\lambda_{n}^{2} h_{v}+m^{2}+m^{4}\right)} \\
\lambda_{n}=\frac{n \pi}{l \sqrt{h_{v}}} ; \mathrm{m}, \mathrm{n}=1,2 \ldots
\end{gathered}
$$

Option 2. Hard fixation of both ends of the shell.

We consider a shell in which the end sections are fixed by flanges, which have great rigidity both in their plane and out of the plane. For the values $\xi=0 ; \xi=1$ the crosssectional contour is not deformed. These fastenings do not permit the movement of the edges of the section along the normal to the middle surface, and also prevent the displacements along the direction of the tangents to the coordinate lines $\mathrm{E}$, and 0 . In addition, rigid fixation prevents the rotation of the tangents to the section.

In this case, the boundary conditions are written as:

$$
\xi=0 \text { and } \xi=1 ; u=v=w=\vartheta_{2}=0
$$

These conditions, expressed in terms of $f_{n}(\xi)$, can be represented: 


$$
\text { at } \xi=0 \text { and } \xi=1 ; f_{n}(0)=f_{n}(l)=0 ; f_{n}^{\prime}(0)=f_{n}^{\prime}(l)=0
$$

The fundamental function corresponding to this type of binding is adopted:

$$
\begin{aligned}
& f_{n}(\xi)=\sin \frac{\lambda_{0}}{l} \xi-\operatorname{sh} \frac{\lambda_{0}}{l}-P_{n}\left(\cos \frac{\lambda_{0}}{l} \xi-\operatorname{ch} \frac{\lambda_{0}}{l} \xi\right), \\
& \text { When } P_{n}=\frac{\operatorname{sh} \lambda_{0}-\sin \lambda_{0}}{\operatorname{ch} \lambda_{0}-\cos \lambda_{0}}, \text { at } \lambda_{0}=\frac{2 n+1}{2} \pi
\end{aligned}
$$

Integral expressions with allowance for this type of binding will be written in the form:

$$
\begin{gathered}
I_{n n}=\int_{0}^{l} f_{n}(\xi) f_{n}(\xi) d \xi=\int_{0}^{l} \sin ^{2} \frac{\lambda_{0}}{l} \xi d \xi \\
I_{n n}^{I V}=\int_{0}^{l} f_{n}(\xi) f_{n}^{I V}(\xi) d \xi=\frac{\lambda_{0}^{4}}{l^{4}} \int_{0}^{l} \sin ^{2} \frac{\lambda_{0}}{l} \xi d \xi, \\
I_{n n}^{n}=\int_{0}^{l} f_{n}(\xi) f_{n}^{\prime \prime}(\xi) d \xi=-\frac{\lambda_{0}^{2}}{l^{2}} \int_{0}^{l} \sin ^{2} \frac{\lambda_{0}}{l} \xi d \xi=-\frac{\lambda_{0}^{2}}{l^{2}} I_{n n}^{*} I_{n n}^{*}=\int_{0}^{l} f_{n}(\xi) f_{n}^{*}(\xi) d \xi \\
I_{\text {in }}=\int_{0}^{l} f_{i}(\xi) f_{n}(\xi) d \xi \approx 0, \quad I_{i n}^{\prime \prime}=-\frac{\lambda_{0}^{2}}{l^{2}} \int_{0}^{l} f_{i}(\xi) f_{n}^{*}(\xi) d \xi \approx 0 \text { at } i \neq n .
\end{gathered}
$$

Substituting (10) into (4) with allowance for (13), after the transformations, we obtain the expression for the square of the circular frequency of the free oscillations of the shell, first dividing the numerator and denominator by the parameter $h_{v}^{2}$ :

$$
\begin{gathered}
W_{\min }^{2}=\frac{\left(1+\frac{1}{2 n}\right)^{4} \lambda_{n}^{4}+m^{4}\left(m^{2}-1\right)\left(m^{2}-1+p^{*}\right)-\left(1+\frac{1}{2 n}\right)^{2} \lambda_{n}^{2} m^{4} 2 \varepsilon_{0}^{*}}{R h \rho^{*}\left[\left(1+\frac{1}{2 n}\right)^{2} \lambda_{n}^{2} \delta_{\left.n n^{h_{v}}+m^{2}+m^{4}\right]}\right.} \\
\text { When } \lambda_{n}=\frac{n \pi}{l \sqrt{h_{v}}}, l=\frac{L}{R}, \delta_{n n}=\frac{I_{n n}^{*}}{I_{n n}}
\end{gathered}
$$

Variant 3. A shell with hinged support on the left end for $\xi=0$ and a clamped right end for $\xi=1$.

The boundary conditions are written as:

$$
f_{n}(\xi)=\left.f_{n}^{\prime \prime}(\xi)\right|_{\xi=0}=0, \quad f_{n}(\xi)=\left.f_{n}^{\prime}(\xi)\right|_{\xi=l}=0
$$

This condition for fixing the end sections of the shell corresponds to the function:

$$
f_{n}(\xi)=\sin \frac{\lambda_{0}}{l} \xi-\frac{\sin \lambda_{0}}{\operatorname{sh} \lambda_{0}} \operatorname{sh} \frac{\lambda_{0}}{l} \xi, \text { when } \lambda_{0}=\frac{4 n+1}{4} \pi
$$

The second and fourth derivatives of the fundamental function can be written in the form:

$$
\begin{gathered}
f_{n}^{\prime \prime}(\xi)=-\frac{\lambda_{0}^{2}}{l^{2}}\left[\sin \frac{\lambda_{0}}{l} \xi+\frac{\sin \lambda_{0}}{\operatorname{sh} \lambda_{0}} \operatorname{sh} \frac{\lambda_{0}}{l} \xi\right]=-\frac{\lambda_{0}^{2}}{l^{2}} f_{n}^{*}(\xi), \\
\operatorname{when} f_{n}^{*}(\xi)=\left[\sin \frac{\lambda_{0}}{l} \xi+\frac{\sin \lambda_{0}}{\operatorname{sh} \lambda_{0}} \operatorname{sh} \frac{\lambda_{0}}{l} \xi\right], \\
f_{n}^{I V}(\xi)=-\frac{\lambda_{0}^{4}}{l^{4}}\left[\sin \frac{\lambda_{0}}{l} \xi+\frac{\sin \lambda_{0}}{\operatorname{sh} \lambda_{0}} \operatorname{sh} \frac{\lambda_{0}}{l} \xi\right]=-\frac{\lambda_{0}^{4}}{l^{4}} f_{n}^{*}(\xi) .
\end{gathered}
$$

Integral expressions for $\mathrm{i}=\mathrm{n}$, we represent: 


$$
\begin{gathered}
I_{n n}=\int_{0}^{l} f_{n}(\xi) f_{n}(\xi) d \xi \\
I_{n n}^{I V}=\frac{\lambda_{0}^{4}}{l^{4}} \int_{0}^{l} f_{n}(\xi) f_{n}(\xi) d \xi=\frac{\lambda_{0}^{4}}{l^{4}} I_{n n} \\
I_{n n}^{n}=\int_{0}^{l} f_{n}(\xi) f_{n}^{n}(\xi) d \xi=-\frac{\lambda_{0}^{2}}{l^{2}} \int_{0}^{l} f_{n}(\xi) f_{n}^{*}(\xi) d \xi=-\frac{\lambda_{0}^{2}}{l^{2}} I_{n n}^{*} \text { when } I_{n n}^{*}=\int_{0}^{l} f_{n}(\xi) f_{n}^{*}(\xi) d \xi \\
I_{\text {in }}=\int_{0}^{l} f_{i}(\xi) f_{n}(\xi) d \xi \approx 0, I_{i n}^{n}=-\frac{\lambda_{0}^{2}}{l^{2}} \int_{0}^{l} f_{i}(\xi) f_{n}^{*}(\xi) d \xi \approx 0 \text { at } i \neq n .
\end{gathered}
$$

Substituting (15) into (4) with regard to (18), after the transformations, we obtain the expression for the square of the circular frequency of free oscillations:

$$
w_{\min }^{2}=\frac{\left(1+\frac{1}{4 n}\right)^{4} \lambda_{n}^{4}+m^{4}\left(m^{2}-1\right)\left(m^{2}-1+p^{*}\right)-\left(1+\frac{1}{4 n}\right)^{2} \lambda_{n}^{2} m^{4} 2 \varepsilon_{0}^{*} \delta_{n n}}{R h \rho^{*}\left[\left(1+\frac{1}{4 n}\right)^{2} \lambda_{n}^{2} \delta_{n n} h_{v}+m^{2}+m^{4}\right]}
$$

When $\lambda_{n}=\frac{n \pi}{l \sqrt{h_{v}}}, l=\frac{L}{R}$

In order to see the difference in the operation of pipelines with different fastenings, we perform a series of calculations using these formulas and analyze the results obtained.

\begin{tabular}{|c|c|c|c|c|c|c|c|c|c|}
\hline \multirow{3}{*}{$\begin{array}{c}\text { Support } \\
\\
P\end{array}$} & \multicolumn{9}{|c|}{$\mathrm{n}=1 ; \mathrm{p}=3 \mathrm{MPa} ; \mathrm{h} / \mathrm{r}=1 / 40(15 \mathrm{x} 1220) ; \mathrm{L} / \mathrm{R}=10$} \\
\hline & \multicolumn{3}{|c|}{ Hinge - hinge } & \multicolumn{3}{|c|}{ hard sealing - hard sealing } & \multicolumn{3}{|c|}{ hard sealing - hinge } \\
\hline & $\mathrm{m}=1$ & $\mathrm{~m}=2$ & $\mathrm{~m}=3$ & $\mathrm{~m}=1$ & $\mathrm{~m}=2$ & $\mathrm{~m}=3$ & $\mathrm{~m}=1$ & $\mathrm{~m}=2$ & $\mathrm{~m}=3$ \\
\hline 0 & 89,68 & 56,70 & 103,91 & 222,52 & 82,17 & 107,73 & 150,46 & 66,99 & 105,36 \\
\hline 0,1 & 89,34 & 55,80 & 105,35 & 222,36 & 81,54 & 107,19 & 150,22 & 66,21 & 104,81 \\
\hline 0,3 & 88,66 & 53,95 & 102,24 & 222,03 & 80,26 & 106,12 & 149,76 & 64,64 & 103,71 \\
\hline 0,5 & 87,97 & 52,04 & 101,11 & 221,69 & 78,96 & 105,03 & 149,29 & 63,03 & 102,6 \\
\hline 0,6 & 87,62 & 51,05 & 100,55 & 221,53 & 78,3 & 104,48 & 149,05 & 62,21 & 102,04 \\
\hline 0,7 & 87,28 & 50,05 & 99,97 & 221,36 & 77,64 & 103,93 & 148,82 & 61,38 & 101,47 \\
\hline
\end{tabular}

The solutions obtained by the formula $(9,14,19)$ are summarized in tables $1-4$ and are illustrated in the form of graphs in Figures 1-4.

Table 1. Dependence of the frequencies of free bending vibrations $\omega_{21}(\mathrm{~Hz})$ of gas pipelines on the parameter of the longitudinal compressive force $\mathrm{P}$ and the method of fixing the pipeline. 


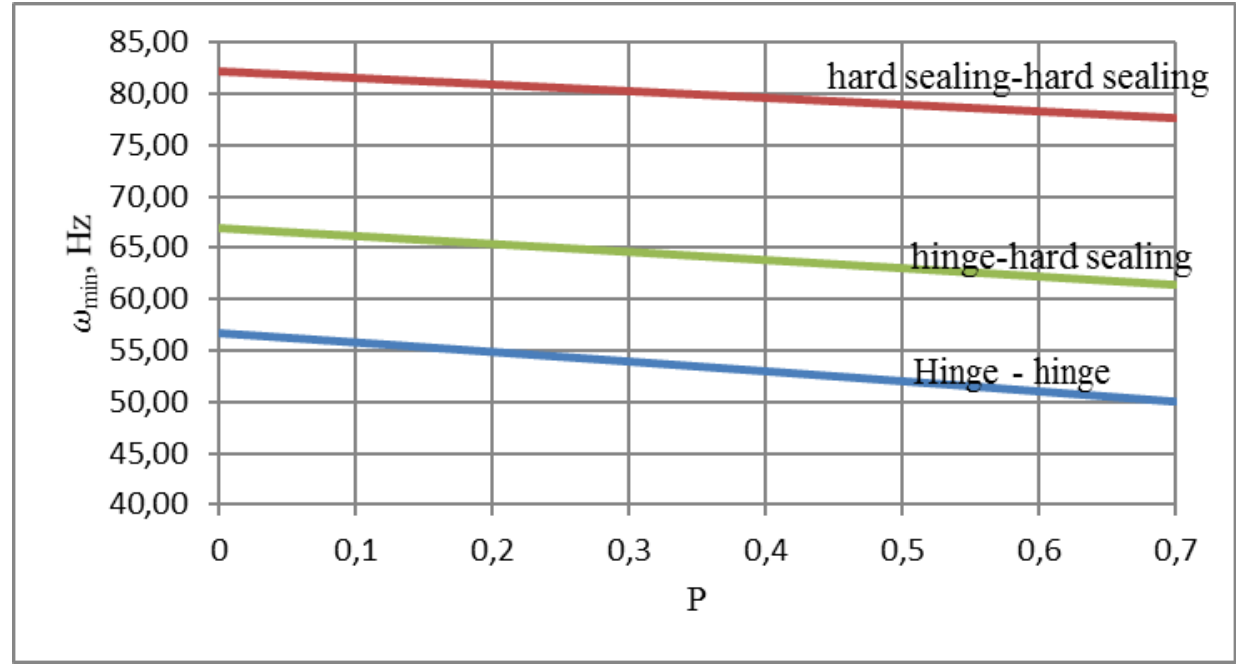

Fig. 5. Dependence of the frequencies of free bending vibrations $\omega_{21}(\mathrm{~Hz})$ of gas pipelines on the parameter of the longitudinal compressive force and the fixing method.

Analysis of the graph in Fig. 5 showed that the change in the parameter of the longitudinal compressive force significantly affects the natural frequencies of the oscillations of the pipeline, reducing them. This effect is more significant on pipelines with hinged support at both ends of the shell, for example, when the parameter of the longitudinal compressive force $\mathrm{P}$ varies from 0 to 0.7 , the frequency decrease $\omega_{21}$ is $12 \%$. For the variant with rigid fixation of both ends, the frequency variation $\omega_{21}$ was $6 \%$, and for hard fastening on one side and hinged on the other, $\omega_{21}$ frequency decreased by $8 \%$.

Differences in growth trends for different fixing methods can be explained by the fact that rigid fixation of the ends of the shell takes over the forces of transverse compression, which reduces the deformation of the pipeline and as a result reduces the effect of the parameter of the longitudinal compressive force on the oscillation frequencies.

Table 2. Dependence of the frequencies of free bending vibrations $\omega_{21}(\mathrm{~Hz})$ of gas pipelines on the internal operating pressure 0 and the method of fixing the pipeline.

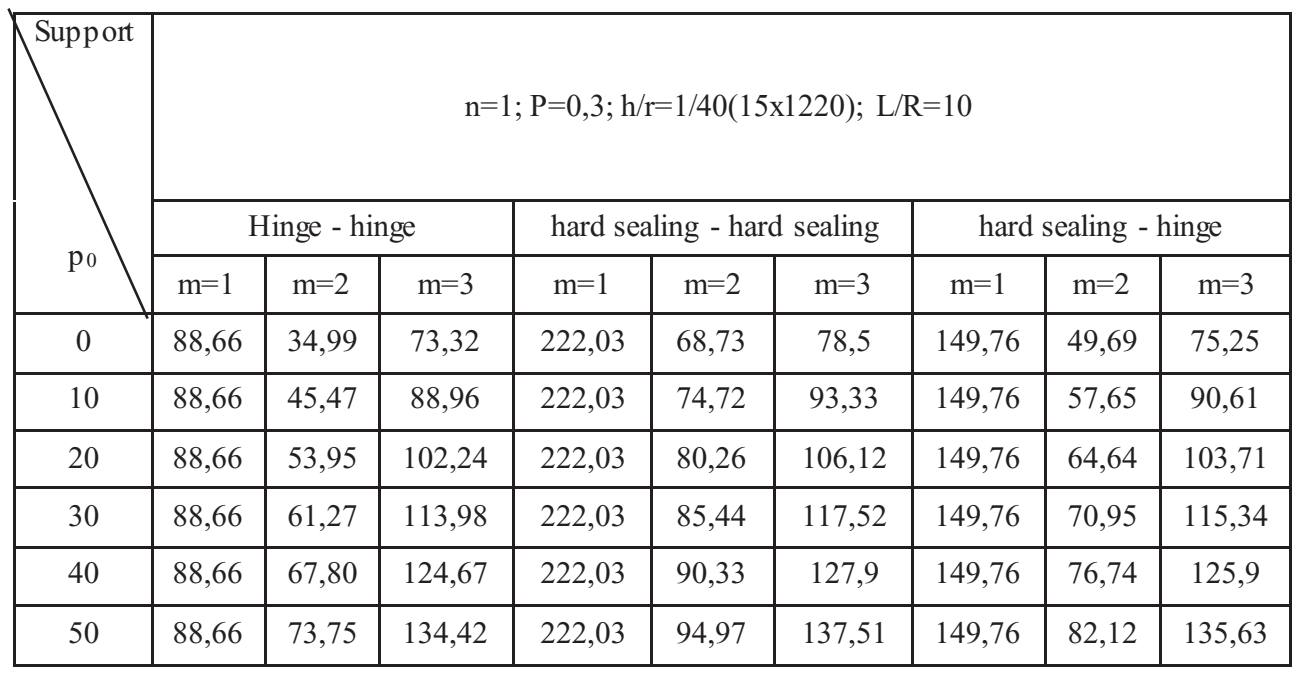




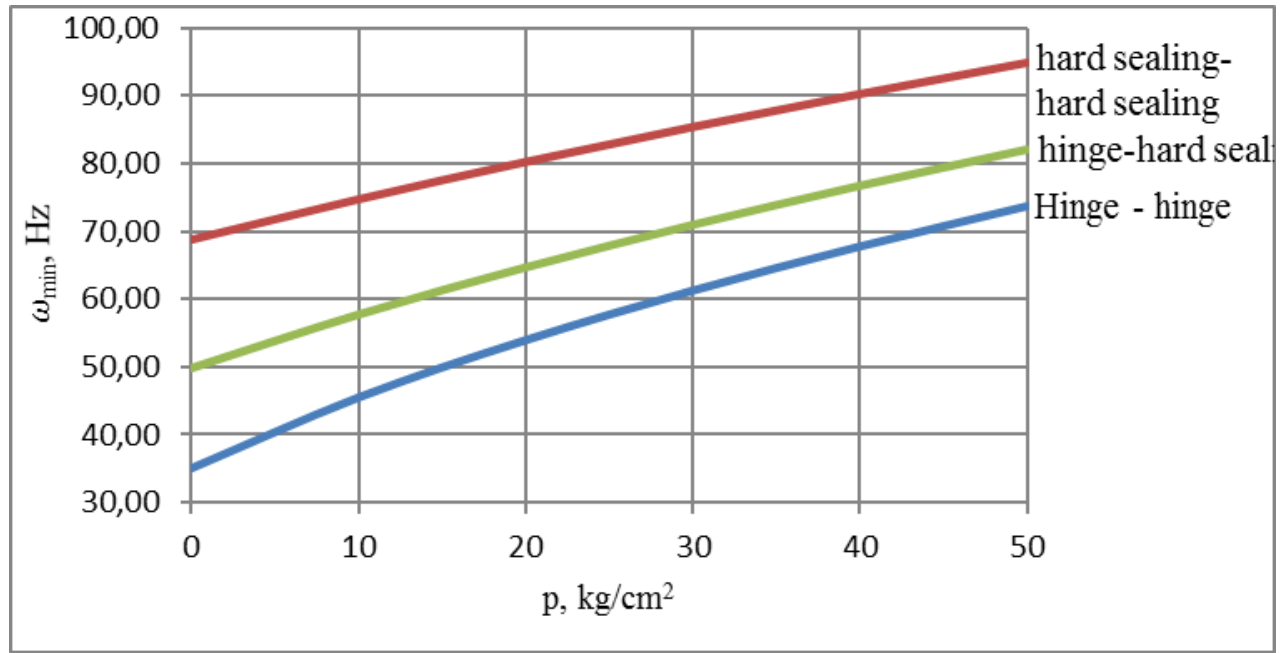

Fig. 6. Dependence of frequencies of free bending vibrations $\omega_{21}(\mathrm{~Hz})$ of gas pip elines on internal working pressure and fixing method.

Analysis of the graph in Fig. 6 showed that a change in the internal operating pressure significantly affects the natural frequencies of the oscillations of the pipeline, increasing them. This effect is more significant on pipelines with a hinged support at both ends of the shell, for example, when the internal operating pressure $\mathrm{p}_{0}$ varies from $0 \mathrm{~kg} / \mathrm{cm} 2$ to $50 \mathrm{~kg} /$ $\mathrm{cm}^{2}$, the frequency increase $\omega_{21}$ is $111 \%$. For the variant with rigid fixation of both ends, the frequency change $\omega_{21}$ was $38 \%$, and for hard fastening on one side and hinged on the other, the frequency $\omega_{21}$ increased by $65 \%$.

Differences in growth trends for different fixing methods can be explained by the fact that when rigidly supporting the ends of the shell on the supports, the rigidity of the pipe increases substantially, and therefore the internal working pressure does not exert such an influence as when hinged.

Table 3. Dependence of the frequencies of free bending vibrations $\omega_{21}(\mathrm{~Hz})$ of gas pipelines on the internal operating pressure 0 and the method of fixing the pipeline.

\begin{tabular}{|c|c|c|c|c|c|c|c|c|c|}
\hline Suppoit & & & & $\mathrm{P}=0,3 ; 1$ & $=1 / 40(15 \mathrm{x}$ & $20) ; p=31$ & & & \\
\hline $\mathrm{I} / \mathrm{Pl}$ & \multicolumn{3}{|c|}{ Hinge - hinge } & \multicolumn{3}{|c|}{ hard sealing - hard sealing } & \multicolumn{3}{|c|}{ hard sealing - hinge } \\
\hline & $\mathrm{m}=1$ & $\mathrm{~m}=2$ & $\mathrm{~m}=3$ & $\mathrm{~m}=1$ & $\mathrm{~m}=2$ & $\mathrm{~m}=3$ & $\mathrm{~m}=1$ & $\mathrm{~m}=2$ & $\mathrm{~m}=3$ \\
\hline 7 & 177,71 & 77,87 & 114,94 & 496,61 & 144,88 & 128,52 & 323,44 & 106,77 & 120,11 \\
\hline 8 & 137,30 & 69,14 & 114,28 & 363,86 & 116,08 & 122,53 & 241,19 & 88,87 & 117,41 \\
\hline 10 & 88,66 & 61,27 & 113,98 & 222,03 & 85,44 & 117,52 & 149,76 & 70,95 & 115,34 \\
\hline 11 & 73,39 & 59,52 & 113,99 & 180,93 & 77,24 & 116,45 & 122,6 & 66,49 & 114,95 \\
\hline 13 & 52,55 & 57,78 & 114,09 & 127,11 & 67,72 & 115,39 & 86,6 & 61,6 & 114,61 \\
\hline 15 & 39,35 & 57,08 & 114,19 & 94,32 & 62,98 & 114,96 & 64,42 & 59,32 & 114,51 \\
\hline
\end{tabular}




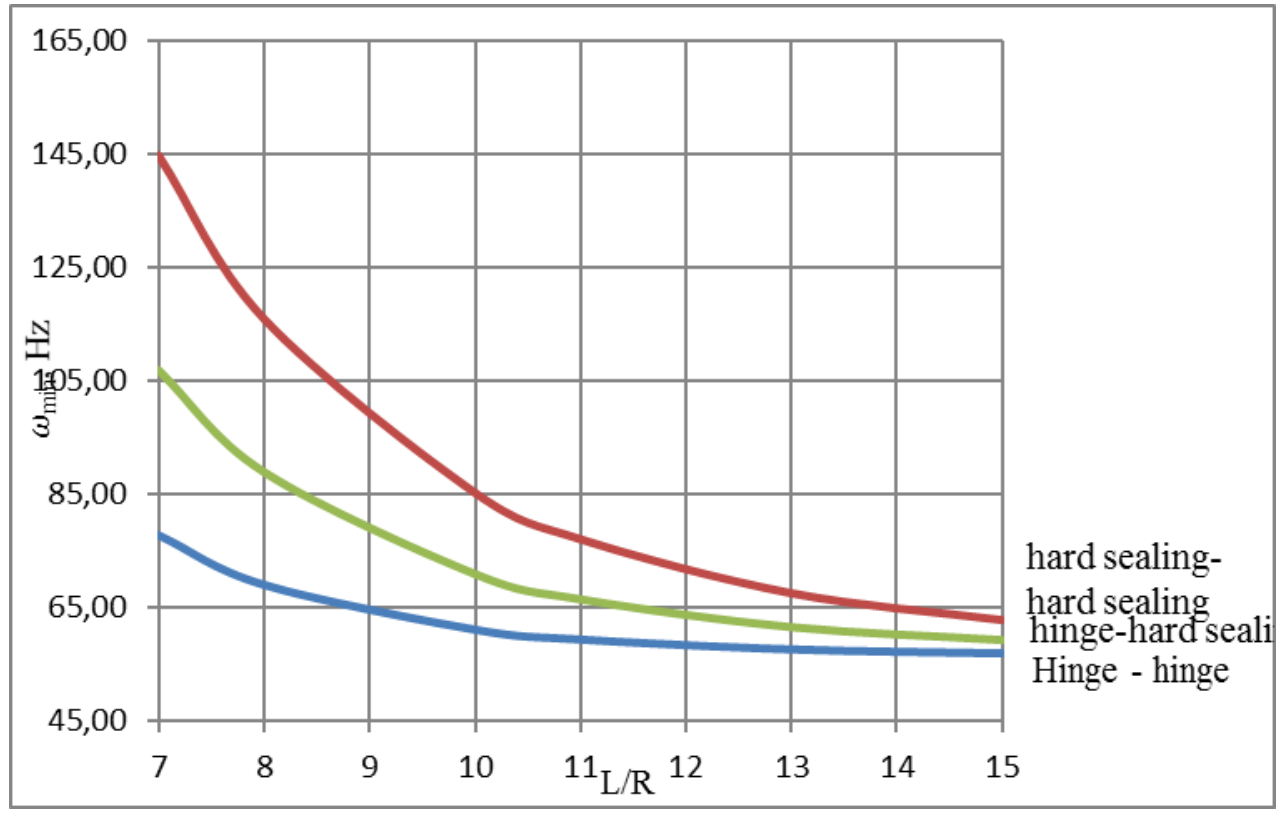

Fig. 7. Dependence of frequencies of free bending vibrations $\omega_{21}(\mathrm{~Hz})$ of gas pipelines on the distance between supports and the method of fixing.

Analysis of the graph in Fig. 7 showed that the change in the distance between supports significantly affects the natural frequencies of the oscillations of the pipeline, reducing them. This effect is more significant on pipelines with a rigid support at both ends of the shell, for example, a change in the ratio L/ R from 7 to 15 , the decrease in the frequencies $\omega_{21}$ is $57 \%$. For the variant with hinged fixing of both ends, the frequency change $\omega_{21}$ was $27 \%$, and for hard fastening on one side and hinged on the other, the frequency $\omega_{21}$ decreased by $44 \%$.

Differences in growth trends for different methods of fixing can be explained by the fact that for short pipes the boundary conditions have a greater effect on the constraint of the deformation of the cross sections, as a result of which the rigidity of these pipes increases with the clamped ends. Therefore, as the length is increased, the frequency variation of the rigidly fixed pipes is more significant.

\section{Conclusion}

Based on these results, we can draw the following conclusions:

- The internal operating pressure, the longitudinal compressive force parameter and the length between the fastenings significantly influence the natural frequencies of the free oscillations of the pipeline, this effect is most noticeable for hinged pipelines;

- An increase in the longitudinal compressive force parameter has a lesser effect on rigidly fixed pipelines ( $6 \%$ reduction);

- The increase in internal operating pressure has a lesser effect on rigidly fixed pipelines (38\% increase) while for hinged $111 \%$ and $65 \%$ for mixed;

- Rigid pinching of pipe ends restricts the deformation of the cross sections, which increases the rigidity of the pipe, but as the distance between the pipeline supports increases, the effect of this constraint becomes small.

A more detailed study of the results obtained will be carried out in the future work. 
The corresponding results in this article were obtained during the execution of the state task of the Ministry of Education and Science of Russia 7.4794.2017/8.9.

\section{References}

1. E.L. Axelrad, V.P. Il'yin, Raschet truboprovodov (Mashinostroenie, 1972)

2. F.M. Mustafin, Sposoby prokladki truboprovodov s primeneniyem opryskivaniya osobo obrabotannymi pochvami 1 (2003)

3. V.P. Il'in, V.G. Sokolov, Industrial and Civil building 12 (2009)

4. V.G. Sokolov, A.V. Bereznev, Izv. universities. Oil and gas 1 (2005)

5. D. Barsi, A. Perrone, Y. Qu, L. Ratto, G. Ricci, V. Sergeev, P. Zunino, Journal of Thermal Science 27, 259-269 (2018)

6. J. Kaikko, A. Mankonen, E. Vakkilainen, V. Sergeev, Energy Procedia 120, 572-579 (2017) 